\title{
REPRODUCTION NATURELLE AMÉNAGÉE \\ DU BROCHET EN PETITS ÉTANGS : ASPECTS ÉCONOMIQUES DE LA PRODUCTION DE JUVÉNILES
}

\author{
J. MANELPHE, C. BRY
}

I.N.R.A., Laboratoire de Génétique des Poissons - 78350 JOUY-EN-JOSAS

\section{RÉSUMÉ}

La production de brochetons de iepeuplement par la méthode de reproduction naturelle aménagée (R.N.A.) fait ici l'objet d'une évaluation économique, basée sur l'étude de quatre ésocicultures.

Celle-ci fait apparaitre les résultats suivants:

- le coût de production (somme des charges d'exploitation et des charges financières) varie de 12.500 à 29.000 F 1986/ha de terrain/an (moyenne : 19.000 F 1986/ha de terrain/an). Cette amplitude de variation est le reflet des différences inter-sites de localisation, de superficie et de nature du terrain, de choix de plan d'aménagement et des matériaux de construction, et de coût des services extérieurs;

- ie prix de revient du brocheton, fonction du coût de production et du niveau de production de chaque exploitation, varie de 1,4 à $2,0 \mathrm{~F}$ (moyenne : $1,7 \mathrm{~F}$ ). En considérant un prix de vente moyen du brocheton de $7 / 8 \mathrm{~cm}$ de $3,5 \mathrm{~F} \mathrm{H}$.T. livré, en 1986, le bénéfice brut dégagé par brocheton est de 1,5 à $2,1 \mathrm{~F}$, selon les sites (moyenne : 1,8 F);

- le produit d'exploitation (total des recettes de vente des juvéniles) varie de 24.000 à 50.000 F 1986/ha de terrain/an (moyenne : $39.500 \mathrm{~F}$ 1986/ha de terrain/an), selon la part de terrain relative aux étangs proprement dits (de 46 à $71 \%$ selon les sites) et le niveau de production de chaque pisciculture (de 1,4 à 2,8 brochetons produits $/ \mathrm{m}^{2}$ d'étang);

- le revenu agricole (produit d'exploitation diminué des charges réelles totales) s'élève en

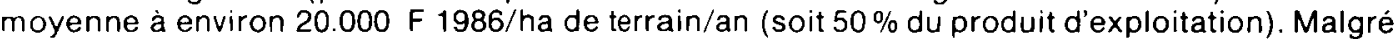
la variabilité de ce paramètre (de 11.000 à $27.000 \mathrm{~F} \mathrm{1986/ha} \mathrm{de} \mathrm{terrain/an,} \mathrm{selon} \mathrm{les} \mathrm{ésocicul-}$ tures), la rentabilité de la R.N.A. demeure très élevée (revenu agricole $=40$ à $60 \%$ du produit d'exploitation).

La présente analyse économique révèle que la méthode de reproduction naturelle aménagée du brochet peut constituer, à l'échelle de quelques hectares :

- pour les exploitants agricoles, un élément intéressant de diversification et une source de revenus complémentaires;

- pour les Fédérations Départementales de Pêche et de Pisciculture et pour certains pisciculteurs en étangs, un moyen simple de produire des brochetons de repeuplement de qualité à un prix de revient particulièrement peu élevé.

\section{MANAGED NATURAL SPAWNING OF PIKE (ESOX LUCIUS L.) IN SMALL PONDS : ECONOMIC ASPECTS OF JUVENILE PRODUCTION}

\section{ABSTRACT}

The production of pike juveniles by the method of managed natural spawning (M.N.S., or R.N.A. in the french text) for the purpose of stocking natural waters was subjected to an economic evaluation based on the study of four pike farms.

This investigation yielded the following results:

- the production cost (i.e. the sum of operating expenses and financial charges) varies between 12500 and 29000 F.F. 1986 per hectare of land per year (average : 19000 F.F. 1986/ha/year). Such a variability results from differences in farm localization and surface, land nature, pond design, building materials and external services costs;

- the prime cost of a pike juvenile ranges from 1.4 to 2.0 F.F. (average: 1.7 F.F.), according to the cost and level of production. Based on an average selling price of $3.5 \mathrm{~F}$.F. per $7 / 8 \mathrm{~cm}$ pike juvenile, the gross profit varies between 1.5 and 2.1 F.F. 1986 per juvenile (average : 1.8 F.F.); 
- the net sales (i.e. the turn-over) ranges from 24000 to 50000 F.F. 1986/ha of land/year (average : 39500 F.F./ha/year), depending on the ratio between pond surface and total surface (range : 46-71\%, according to the site) and the production level of each farm (range: $1.4-2.8$ juvenile per $\mathrm{m}^{2}$ of pond) ;

- the average farm income (i.e. the net sales minus total costs) amounts to 20000 F.F. 1986/ha of land/year (i.e. $50 \%$ of the net sales). Despite the variability of this parameter (range: 1100027000 F.F. 1986/ha of land/year), the profitability of pike juvenile production from M.N.S. remains very high, with a farm income amounting to $40-60 \%$ of the net sales.

The present economic analysis demonstrates that the production of pike juveniles from M.N.S., practised at the scale of a few hectares, may represent:

- for farmers, an attractive factor of diversification and a source of complementary income

- for the local Angling and Fish Culture Associations and for some pond fish culturists, a simple and economical method of producing quality pike juveniles for stocking natural waters.

\section{INTRODUCTION}

La reproduction naturelle aménagée (R.N.A.) du brochet (Esox lucius L.) est une méthode de reproduction-grossissement permettant, à l'aide de peu de moyens humains et techniques, de produire des brochetons destinés à être libérés dans le milieu naturel (poissons de repeuplement).

Elle se pratique dans un milieu semi-naturel favorable à la fraie ainsi qu'à la survie et à la croissance des jeunes stades, et se prêtant à une récolte efficace des juvéniles produits : il s'agit d'étangs vidangeables de faible superficie (400 à $\left.3.000 \mathrm{~m}^{2}\right)$, peu profonds, généralement pourvus d'une végétation herbacée développée, dotés d'un dispositif de piégeage à l'arrivée d'eau et de niveau d'eau stable.

Dans chacun de ces étangs, on introduit, quelque temps avant le début de la période de reproduction, un brochet femelle (de taille adaptée aux dimensions de l'étang) et deux mâles, ainsi que du poisson-fourrage de taille adéquate. La récolte des brochetons a généralement lieu au courant du mois de mai.

Depuis 1980, la R.N.A. est expérimentée à l'ésociculture du Paraclet (Somme). Les premiers résultats, obtenus de 1980 à 1982, au Paraclet et dans la Dombes (Ain), font état d'une production moyenne de l'ordre de 2 brochetons de taille 7 à $8 \mathrm{~cm}$ (poids moyen voisin de $2 \mathrm{~g}$ ) par $\mathrm{m}^{2}$ de surface en eau (Bry et Souchon, 1982; Bry et al., 1983; Bry et al., 1984).

Ces résultats ont, par la suite, été confirmés, tant au Paraclet, de 1983 à 1987, que dans plusieurs autres ésocicultures de différents départements.

La R.N.A. s'est ainsi avérée reproductible à la fois dans le temps et dans l'espace, et fait actuellement l'objet d'une mise en ceuvre progressive au sein de plusieurs exploitations ésocicoles de création récente. Aussi nous a-t-il paru opportun d'aborder dans le présent article l'étude de cette technique sous l'angle économique.

\section{MATÉRIEL ET MÉTHODES}

La présente évaluation économique de la R.N.A. est basée sur les éléments techniques, économiques et financiers de quatre ésocicultures:

- le Centre du Paraclet, appartenant au Conseil Supérieur de la Pêche, installé à proximité d'Amiens (Somme);

- une pisciculture fédérale du centre de la France (P.Féd.1);

- une pisciculture fédérale de l'ouest de la France (P.Féd.2);

- une pisciculture privée (P.Pr.), intégrée à une exploitation agricole traditionnelle, située dans la vallée de la Noye (Somme).

Elle prend également en compte les renseignements recueillis auprès de professionnels de différents secteurs d'activité (pêche, pisciculture, construction d'étangs, économie et gestion agricoles, fiscalité, etc...)

A partir des données ainsi rassemblées, plusieurs paramètres économiques sont mesurès et exprimés en francs $1986^{*}$, dans le but d'apprécier la rentabilité actuelle de la R.N.A. :

\footnotetext{
"Les déflateurs utilisés (indices de formation brute de capital fixe des Sociètés et Quasi-Sociétés et des Entreprises industrielles, et indices des prix des consommations intermédiaires, dans le Domaine de l'Agriculture, de la Sylviculture et de la Pêche) sont issus de l'Extrait du Rapport sur les Comptes de la Nation, pour l'année 1986, de l'I.N.S.E.E.
} 
- le coût de production, égal à la somme des charges d'exploitation et des charges financières, duquel découle le prix de revient du brocheton;

- le produit d'exploitation (= chiffre d'affaires);

- le résultat d'exploitation, égal au produit d'exploitation diminué des charges d'exploitation;

- le revenu agricole, égal au résultat d'exploitation diminué des charges financières, qui traduit la "rentabilité" de la R.N.A.

\section{Charges d'exploitation}

Les charges d'exploitation comprennent les frais d'empoissonnement, les frais de personnel, les dotations aux amortissements et quelques autres charges.

\subsection{Frais d'empoissonnement}

Ils correspondent à l'achat des géniteurs-brochets et du poisson-fourrage. Ceux-ci sont obtenus auprès de pisciculteurs en étangs ou de particuliers propriétaires d'étangs, au moment des vidanges, généralement annuelles, entre novembre et février.

Deux cas de figure sont envisageables :

- les poissons sont achetés entre novembre et janvier et stockés après calibrage dans un ou plusieurs étangs de production (sous réserve d'une profondeur suffisante); à la mi-février, ils sont récupérés par vidange et répartis sur l'ensemble des étangs;

- les poissons sont achetés en février et directement répartis entre les différents étangs de production, à condition de limiter leur période de captivité au seul transport.

Le créneau de mise en charge optimum (poids en février de la femelle destinée à un étang de superficie donnée) peut varier quelque peu selon le lieu et le type d'étang utilisé (Bry et al., 1984). Par souci de simplification, on considère ici les valeurs indicatives d'une femelle de $1,75 \mathrm{~kg}$ et de deux mâles de $1 \mathrm{~kg}$ pour un étang de $1.500 \mathrm{~m}^{2}$, et on les applique aux étangs d'autres dimensions proportionnellement à leur surface.

Dans l'hypothèse d'une acquisition des brochets en novembre, la quantité de poissonfourrage nécessaire tient compte de l'entretien des géniteurs durant le stockage hivernal, de novembre à février (soit en moyenne $0,5 \mathrm{~kg}$ de poisson-fourrage pour $1 \mathrm{~kg}$ de brochet), ainsi que pendant et après la saison de reproduction, de mars à mai (soit $0,6 \mathrm{~kg}$ de poisson-fourrage pour $1 \mathrm{~kg}$ de brochet, dans des conditions thermiques moyennes en avril-mai):

Les frais d'empoissonnement sont évalués sur les bases suivantes:

- acquisition des poissons en novembre ;

- biomasse de brochets nécessaire égale à $30 \mathrm{~kg}$ par hectare d'étangs (soit $25 \mathrm{~kg} / \mathrm{ha}$ d'étangs + marge de sécurité de $20 \%$ );

- biomasse de poisson-fourrage légèrement excédentaire : $40 \mathrm{~kg}$ par hectare d'étangs ;

- prix moyens d'achat, sur place, en 1986, de $50 \mathrm{FH}$.T./kg pour les brochets et de $25 \mathrm{FH}$.T./kg pour le poisson-fourrage (gardons et/ou rotengles).

N.B. : Le devenir des géniteurs et du poisson-fourrage, récupérés en mai lors de la récolte des brochetons, n'est pas pris en considération dans la présente étude.

\subsection{Frais de personnel}

La R.N.A. fait appel à une main-d'œuvre saisonnière, sans qualification particulière requise, essentiellement mobilisée en mai au moment de la récolte des brochetons (les opérations effectuées pendant chaque saison de production sont détaillées en annexe).

Les frais de personnel sont calculés sur la base d'un salaire horaire ajusté à $30 \mathrm{~F}$ et tiennent compte des charges patronales (32\%).

N.B. : a titre indicatif, le salaire horaire conventionnel minimal d'un ouvrier agricole variait, en 1986, de 26,05 F à 30,64 F, en fonction du coefficient de qualification (l'Action Agricole, avril 1986).

\subsection{Dotations aux amortissements et autres charges}

1.3.1. Amortissements sur installations

Une part des sommes engagées lors de l'aménagement de l'ésociculture est amortissable : il s'agit de celle relative à l'achat et à la pose des tuyaux P.V.C. et des divers ouvrages en béton (ouvrages à la prise d'eau et à la restitution, ouvrages de distribution et boîtes de branchement). On considère un amortissement sur vingt ans de ces éléments.

Les travaux de terrassement constituent, quant à eux, une amélioration permanente du fonds, non amortissable.

\subsubsection{Frais de réaménagement}

Un réaménagement périodique des étangs et des fossés est nécessaire afin de maintenir des conditions d'exploitation satisfaisantes. Ainsi, les étangs doivent-ils être remodelés tous les quinze 
à vingt ans, selon la vitesse d'évolution du milieu (importance des dépôts). Les fossés doivent être recreusés en moyenne tous les cing ans.

Les frais de réaménagement sont évalués en se basant sur le coût d'aménagement enregistré pour chacune des ésocicultures étudiées.

\subsubsection{Frais d'entretien du site}

L'entretien du site consiste en la coupe régulière de la végétation des étangs (durant l'assec, en une seule fois, à la fin de l'été) et des digues (à deux reprises, avant les vidanges de mai et à la fin de l'été).

Le matériel d'entretien retenu pour le calcul des frais est le mème pour les quatre piscicultures: il s'agit d'une tondobroyeuse automotrice permettant le fauchage d'un hectare de ter rain en cinq heures en moyenne.

Les frais d'entretien du site rapportés comprennent l'amortissement (sur cinq ans) et le fonctionnement (dépenses en carburant et en entretien) de la tondobroyeuse.

N.B. : les calculs sont basés sur les données techniques fournies par le fabricant de la tondobroyeuse. Il convient de signaler, par ailleurs, que l'entretien peut être réalisé, sur certains sites, au moyen d'un tracteur équipé d'une machine à fléaux.

\subsubsection{Frais de transport*}

Ces frais recouvrent le transport des brochets et du poisson-fourrage et la livraison des brochetons. Ces opérations, nécessitant l'utilisation d'une installation d'oxygène doivent être effectuées à l'aide d'un véhicule muni d'un plateau, conformément à la législation.

Les frais de transport sont calculés pour un véhicule de puissance fiscale égale à $9 \mathrm{CV}$ (prix de revient au km égal à 1,47 F en 1986, donnée citée dans le Guide pratique du contribuable, 1987).

N.B. : le transport de brochetons conditionnés en sacs de polyéthylène gonflés à l'oxygène peut être réalisé au moyen de n'importe quel véhicule.

1.3.5. Frais en équipements divers*

Ils correspondent à l'achat des différentes fournitures nécessaires à l'équipement du personnel, ainsi qu'à la pêche et au transport des poissons, fournitures faisant l'objet d'un amortissement sur trois, cinq ou dix ans selon les cas.

\subsubsection{Impôt foncier}

Les impôts fonciers indiqués pour le Paraclet et la pisciculture privée étudiée ici sont ceux acquittés en 1986 pour la parcelle aménagée en ésociculture; pour les deux piscicultures fédérales, ce sont ceux acquittés, en 1986, pour chaque exploitation dans sa totalité (ésociculture et parcelles voisines), rapportés à la superficie de l'ésociculture seule.

\section{Charges financières}

Afin de se placer dans le cas d'un particulier ou d'une association créant une ésociculture à I'heu re actuelle, et disposant de capitaux limités, des frais financiers sont calculés pour chacune des piscicultures étudiées: ils correspondent aux intérêts déboursés sur un emprunt bancaire d'un montant égal à $80 \%$ du coût d'investissement exprimé en francs 1986 (celui-ci comprend l'achat đu terrain nu, frais de notaire compris, et les frais d'aménagement de l'ésociculture), au taux de 10,1\% sur quinze ans (taux en vigueur en 1986).

N.B. : le mode de fonctionnement financier des piscicultures fédérales leur évite gènéralement le recours à un emprunt bancaire. Dans le cas de la pisciculture privée étudiée ici, l'investissement modéré réalisé (frais d'aménagement de l'ésociculture uniquement) n'a pas non plus nécessité d'emprunt. Il faut signaler, par ailleurs, l'existence d'avantages financiers pouvant être accordés aux jeunes pisciculteurs, sous certaines conditions d'âge, de nationalité et de capacité professionnelle (dotation d'installation, prêts spéciaux à des taux bonifiés).

\section{Produit d'exploitation}

Le produit d'exploitation est égal au montant total H.T. des recettes de vente des juvéniles.

Il est évalué sur les bases suivantes:

- un niveau de production moyen, établi pour chaque ésociculture d'après les résultats obtenus sur quelques étangs et sur une ou plusieurs années** ;

\footnotetext{
- Par souci d'allégement, les éléments de calcul des frais de transport et des frais en équipements divers ne sont pas détaillés ici; ils peuvent cependant être communiqués sur simple demande.

" "Sur une année et d'après les productions de 2, 3 ou 7 étangs, respectivement pour P.Féd.2, P.Pr. et P.Féd.1. Sur huit années et 17 références pour le Paraclet.
} 
- un prix de vente moyen du brocheton de $7 / 8 \mathrm{~cm}$ de $3.50 \mathrm{~F} \mathrm{H.T.} \mathrm{livré} \mathrm{(moyenne} \mathrm{des} \mathrm{tarifs} \mathrm{pratiqués}$ en 1986 par cinq piscicultures privées).

\section{RÉSULTATS ET DISCUSSION}

\section{Charges d'exploitation}

Les charges d'exploitation représentent en moyenne, sur les quatre ésocicultures étudiées, environ $60 \%$ des charges totales (Figure 1) et moins de $30 \%$ du produit d'exploitation (Figure 2).

Elles varient d'un site à l'autre de 9.000 à $16.000 \mathrm{~F} /$ ha de terrain/an (Tableau II), en fonction des caractéristiques du terrain (situation géographique, superficie, nature du sol) et de l'aménagement réalisé (dimensions des étangs, choix des matériaux de construction, coût des services extérieurs).

Tableau I : Présentation des ésocicultures étudiées.

Table I : Characteristics of the studied pike farms.

\begin{tabular}{lcccc}
\hline Sites & Paraclet & P.Féd.1 & P.Féd.2 & P.Pr. \\
\hline Superficie du terrain (ha) & 2,1 & 6.5 & 2,6 & 1,95 \\
Etangs de production & & & & \\
Nombre & 8 & 18 & 20 & 10 \\
Superficie unitaire (m2) & 1500 à 2000 & 2500 à 3000 & 400 à 900 & 750 à 1750 \\
Superficie totale (ha) & 1,3 & 4.6 & 1,2 & 0.95 \\
S. en eau / S. du terrain (\%) & 62 & 71 & 46 & 49 \\
Niveau de production moyen & & & & \\
Pds moyen des brochetons récoltés (g) & 2,6 & 2,2 & 1.3 & $>3$ \\
Nbre moyen de brochetons produits/m2 & 2,3 & 1,6 & 2,8 & 1,4 \\
\hline
\end{tabular}

\subsection{Frais d'empoissonnement}

Les frais d'empoissonnement forment en moyenne $7 \%$ des charges totales (Figure 1).

Ils sont proportionnels à la surface en eau (2.500 F/ha d'étangs/an, dans l'hypothèse retenue ici) et augmentent donc, pour une superficie d'exploitation donnée, lorsque la superficie relative en eau augmente (Tableaux I et II).

\subsection{Frais de personnel}

Les frais de personnel constituent en moyenne $14 \%$ des charges totales (Figure 1).

IIs varient, selon les sites, de 2.000 à un peu plus de $3.000 \mathrm{~F} /$ ha de terrain/an (Tableau II):

Tableau II : Charges d'exploitation (en francs 1986 H.T./ha de terrain/an).

Table II : Operating expenses (in F.F. 1986/ha of land/year, exclusive of tax).

\begin{tabular}{|c|c|c|c|c|c|}
\hline Sites & Paraclet & P.Fed.1 & P.Féd.2 & P.Pr. & $\begin{array}{l}\text { Moyenne } \\
\text { sur les } \\
4 \text { sites }\end{array}$ \\
\hline Frais d'empoissonnement & 1550 & 1750 & 1150 & 1200 & 1400 \\
\hline Frais de personnel & 2750 & 2000 & 3150 & 2800 & 2700 \\
\hline Amortissements sur installations & 3600 & 2050 & 1150 & 600 & 1850 \\
\hline Frais de réaménagement & 3500 & 1100 & 1000 & 400 & 1500 \\
\hline Frais d'entretien du site & 2100 & 750 & 1700 & 2250 & 1700 \\
\hline Frais de transport & 550 & 450 & 500 & 400 & 450 \\
\hline Frais en équipements divers & 1300 & 650 & 1200 & 1300 & 1100 \\
\hline Impot foncier & 950 & 200 & 100 & 150 & 350 \\
\hline Charges d'exploitation totales & 16300 & 8950 & 9950 & 9100 & 11050 \\
\hline
\end{tabular}


- ils augmentent, pour une superficie d'exploitation donnée (Paraclet, P.Féd.2, P.Pr.), avec le nombre d'étangs aménagés. Par exemple, le temps nécessaire aux contrôles de croissance est proportionnel au nombre de lots; dans une moindre mesure, le temps de recolte augmente avec le nombre de vidanges à effectuer (voir détail des opérations en annexe);

- ils diminuent sensiblement lorsque la superficie de l'exploitation augmente (P.Féd.1).

N.B. : pour le calcul de ces frais, on a considéré que le personnel était uniquement constitué de main-d'œurre salarièe.

\subsection{Dotations aux amortissements et autres charges}

\subsubsection{Amortissements sur installations}

Les dotations aux amortissements sur installations représentent en moyenne $10 \%$ des charges totales (Figure 1).

Elles diffèrent très nettement d'un site à l'autre (de 600 à $3.600 \mathrm{~F} /$ ha de terrain/an; Tableaull), selon l'aménagement réalisé:

- nombre d'étangs aménagés sur une superficie donnée de terrain (nombre de dispositifs d'alimentation et de vidange) ;

- tuyaux P.V.C. : longueurs utilisées, diamètres choisis (à titre indicatif, le coût du mètre-linéaire des tubes et le coût unitaire des raccords varient d'un facteur 1 à 2 ou 3, lorsque le diamètre augmente de 125 à $200 \mathrm{~mm}$, pour une même qualité de tuyau);

- ouvrages en béton : nombre et qualité des ouvrages installés;

- travaux annexes éventuels (par exemple : utilisation d'argile exogène pour parfaire l'étanchéité au niveau des dispositifs de vidange, nécessaire ou non selon la nature du sol).

1.3.2. Frais de réaménagement

Les frais de réaménagement (remodelage des étangs et des fossés) forment en moyenne $8 \%$ des charges totales (Figure 1).

II convient de souligner leur forte variabilité en fonction des paramètres suivants:

- la nature du sol:

- perméabilité (des étangs sont potentiellement d'autant plus sujets aux problèmes d'étanchéité - nécessité de travaux de colmatage - que le sol est pauvre en argile);

- structure (les travaux de terrassement sont techniquement plus difficilement réalisables facturation plus élevée - lorsque le sol est de structure peu stable);

- le plan d'aménagement (superficie relative en étangs, longueur totale en fossès) :

- la conformation des étangs : le coût de réaménagement est d'autant plus élevé que le volume des travaux exécutés dans les étangs mêmes est important par rapport à celui des travaux exécutés depuis la berge) (à ce sujet, l'entretien d'étangs de faible largeur - inférieure à $25 \mathrm{~m}$ peut être en grande partie réalisé depuis la digue et est donc particulièrement économique);

- le devenir des terres extraites : la facturation diffère notablement selon que les terres extraites sont stockées sur le site ou transportées en dehors de celui-ci ;

- l'entreprise exécutant les travaux : la facturation est variable selon le personnel mobilisé. les engins de travaux utilisés, le degré de finition du travail souhaité et, surtout, selon la situation locale du marché de l'offre et de la demande.

Les frais de réaménagement varient ainsi, selon les sites, de 400 a $3.500 \mathrm{~F} / \mathrm{ha}$ de terrain/an (Tableau II). A titre indicatif, le coût d'extraction du $\mathrm{m}^{3}$ de terre varie, selon les ésocicultures étudiées, de 6 à 25 F 1986 H.T. (terrassement uniquement, non compris le travail de finition).

\subsubsection{Frais d'entretien du site}

Les frais d'entretien du site (frais de fonctionnement et amortissement de la tondobroyeuse) constituent en moyenne $9 \%$ des charges totales (Figure 1).

Ils varient, d'un site à l'autre, de 750 à $2.250 \mathrm{~F} /$ ha de terrain/an (Tableau II) et comprennent principalement la dotation aux amortissements (rapportée à l'hectare de terrain), additionnée de frais de fonctionnement peu élevés (de 150 à $250 \mathrm{~F} / \mathrm{ha}$ de terrain/an).

\subsubsection{Frais de transport}

Les frais de transport représentent seulement, en moyenne, un peu plus de $2 \%$ des charges totales (Figure 1). (Tableau II).

Dans l'hypothèse de travail retenue ici, ils s'èlèvent, en moyenne, à $450 \mathrm{~F} / \mathrm{ha}$ de terrain/an

N.B. : Les frais de transport peuvent varier autour de la moyenne indiquée ci-dessus, selon la localisation de l'ésociculture par rapport aux lieux d'achat des reproducteurs et de destination des juvéniles. 


\subsubsection{Frais en équipements divers}

Les frais en équipements divers forment en moyenne $6 \%$ des charges totales (Figure 1).

IIs varient de $650 \mathrm{~F} / \mathrm{ha}$ de terrain/an pour l'exploitation la plus grande (P.Féd.1) à 1.200$1300 \mathrm{~F} / \mathrm{ha}$ de terrain/an, pour les trois autres exploitations (Tableau II).

1.3.6 Impôt foncier

Le montant de l'impôt foncier (basé sur la valeur locative attribuée au terrain) varie, pour un type de terrain donné, d'un département à l'autre et également d'une commune à l'autre. C'est ainsi qu'en Picardie, l'imposition foncière sur un terrain classé en "étang" peut varier de 200 à $1.500 \mathrm{~F}$ par hectare et par an (donnée du Centre des Impôts Fonciers d'Amiens).

L'impôt foncier demeure toutefois une charge d'exploitation mineure: il constitue en moyenne moins de $2 \%$ des charges totales (Figure 1 ).

\section{Charges financières}

Les charges financières sont proportionnelles au montant de l'investissement initial (Tableau III). Celui-ci comprend l'achat du terrain, d'une part, l'aménagement de l'ésociculture, d'autre part:

- le coũt du terrain nu varie, sur les quatre sites étudiés, de 15.000 à $30.000 \mathrm{~F} / \mathrm{ha}$ (frais de notaire non compris). D'une manière plus générale, le coût d'un terrain de type "prairie naturelle" (terrain adapté pour l'installation d'une ésociculture, sous réserve d'une alimentation en eau convenable) varie, en France, de 8.000 à $45.000 \mathrm{~F} / \mathrm{ha}$ (moyenne : $18.000 \mathrm{~F} / \mathrm{ha}$ ), en fonction du marché de l'offre et de la demande (données 1985, citées par Lefebvre, 1987);

- le coût d'aménagement de l'ésociculture varie très sensiblement selon les sites, d'environ 30.000 à près de $210.000 \mathrm{~F} / \mathrm{ha}$ de terrain (moyenne : $120.000 \mathrm{~F} / \mathrm{ha}$ ), sous l'influence de plusieurs facteurs : ceux précédemment invoqués pour expliquer la variabilité des frais de réaménagement (nature du sol, plan d'aménagement, conformation des étangs, devenir des terres extraites, tarifs pratiqués par les entreprises locales), auxquels il faut ajouter la profondeur moyenne des étangs aménagés (cubage de terres extraites) et le choix des matériaux de construction (tuyaux P.V.C., ouvrages en béton).

Tableau III : Charges financières (en francs 1986 H.T./ha de terrain/an).

Table III : Financial charges (in F.F. 1986/ha of land/year, exclusive of tax).

\begin{tabular}{|c|c|c|c|c|c|}
\hline Sites & Paraclet & P.Féd.1 & P.Frd. 2 & P.Pr. & $\begin{array}{l}\text { Moyenne } \\
\text { sur les } \\
4 \text { sites }\end{array}$ \\
\hline $\begin{array}{l}\text { Investissement inftial } \\
\text { (en F } 1986 \mathrm{H} . \mathrm{T} \text {. par ha de terrain) } \\
\text { Achat terrain } \\
\text { Amenagement terrain } \\
\text { Coot total. }\end{array}$ & $\begin{array}{r}38150 \\
208750 \\
246900\end{array}$ & $\begin{array}{rr}18 & 800 \\
127 & 550 \\
146 & 350\end{array}$ & $\begin{array}{r}36800 \\
116900 \\
153700\end{array}$ & $\begin{array}{ll}38 & 400 \\
27 & 900 \\
66 & 300\end{array}$ & $\begin{array}{rr}33 & 050 \\
120 & 250 \\
153 & 300\end{array}$ \\
\hline Charges Inancieres & 12950 & 7700 & 8050 & 3450 & 8050 \\
\hline
\end{tabular}

- Frats de notatre compris

Dans tous les cas, les charges financières sont un poste majeur de charges : elles représentent, en moyenne, environ $40 \%$ des charges totales (Figure 1) et $20 \%$ du produit d'exploitation (Figure 2). 

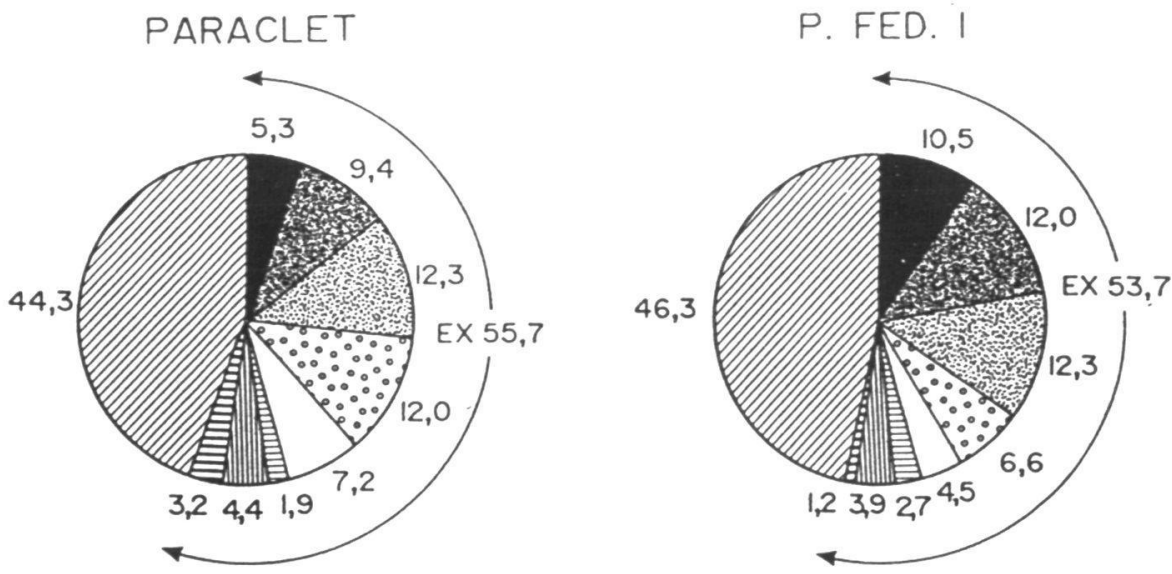

\section{MOYENNE SUR LES}

\section{SITES}
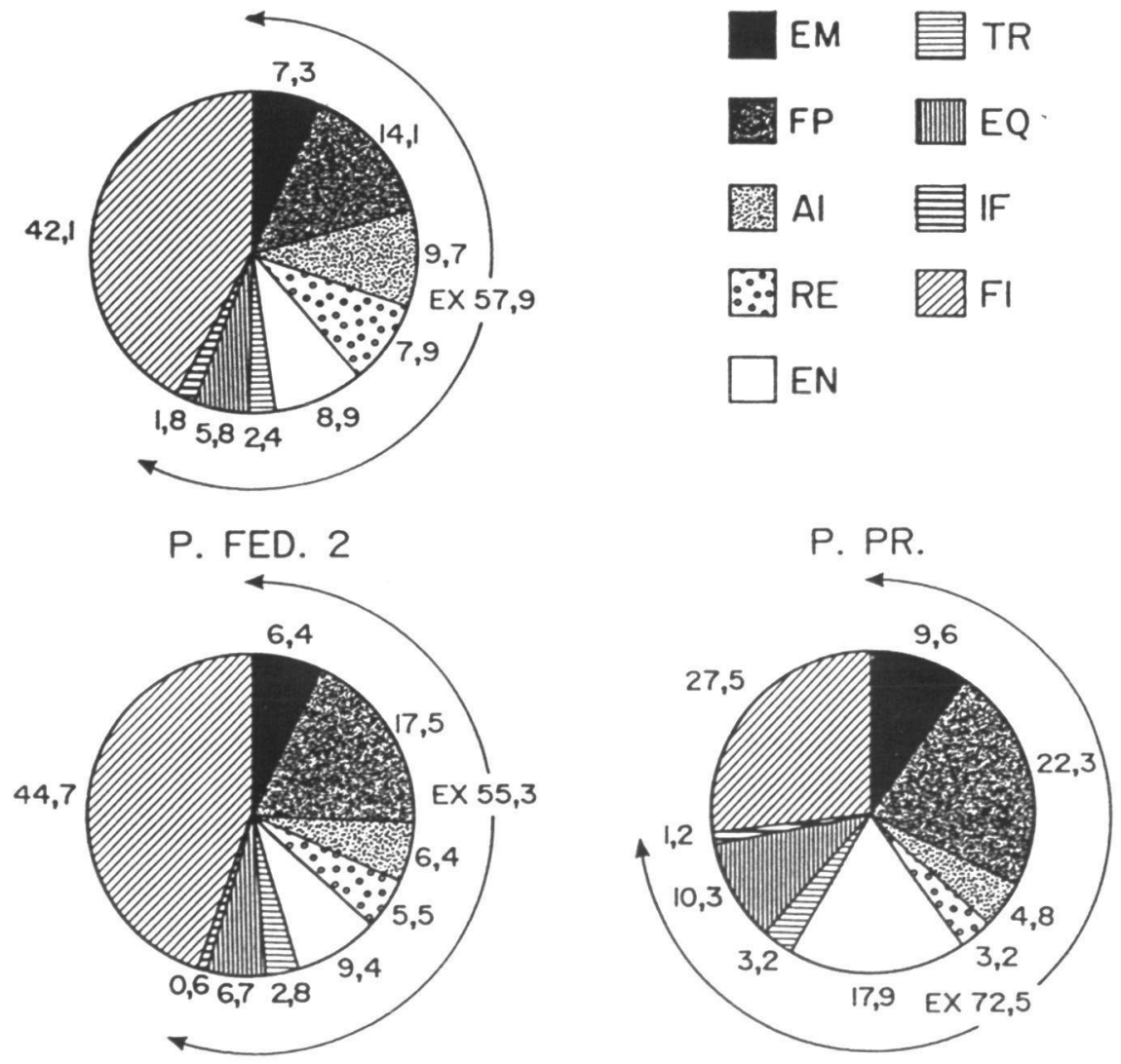

\section{Figure 1 : Charges exprimées en \% des charges totales}

- EM : Frais d'empoissonnement

- FP : Frais de personnel

- Al : Amortissements sur installations

- RE : Frais de réaménagement

- EN : Frais d'entretien du site

$\begin{array}{ll}\text { - TR } & \text { Frais de transport } \\ \text { - EQ } & : \text { Frais en équipements divers } \\ \text { - IF } & : \text { Impót foncier } \\ \text { - EX } & : \text { Charges d'exploitation } \\ \text { - FI } & \text { Charges financières }\end{array}$

\section{Figure 1 : Representation of various expenses in $\%$ of total costs.}

- EM : Fish-stock expenses

- FP : Cost of labour

- Al : Facilities depreciation costs

- RE : Pond rehabilitation costs

- EN : Pond maintenance costs
- TR : Transportation costs

- EQ : Miscellaneous equipment expenses

- IF : Land-tax

- EX : Operating expenses

- FI : Financial charges 


\section{Coût de production et prix de revient du brocheton}

Le coût de production (charges d'exploitation + charges financières) varie, sur les quatre ésocicultures étudiées, de $12.500 \mathrm{~F} /$ ha de terrain/an (enregistré par P.Pr.: coût d'aménagement le plus bas) à $29.000 \mathrm{~F} /$ ha de terrain/an (enregistré par le Paraclet : coût d'aménagement le plus élevé), avec une moyenne d'environ $19.000 \mathrm{~F} /$ ha de terrain/an (Tableau IV).

Le prix de revient du brocheton qui en découle, varie de 1,4 à 2,0 F (moyenne: 1,7 F), d'où un bénéfice brut dégagé de 1,5 à 2,1 $\mathrm{F}$ par brocheton vendu (moyenne : 1,8 F).

N.B. : Si l'on tient compte du fait que les quatre exploitations étudiées n'ont pas contracté d'emprunt bancaire (aucun frais financier), le coût de production se réduit alors aux charges d'exploitation et le prix de revient du brocheton n'est plus que de 0,8 à $1,3 \mathrm{~F}$ (bénéfice brut dégagé par brocheton : 2,2 à 2,7 F).

Tableau IV : Coût de production (en francs 1986 H.T./ha de terrain/an) et prix de revient du brochetor. (en francs 1986 H.T.).

Table IV : Production cost (in F.F. 1986/ha of land/year, exclusive of tax) and prime cost of a pike juvenile (in F.F. 1986, exclusive of tax).

\begin{tabular}{|c|c|c|c|c|c|}
\hline Sites & Paraclet & P.Féd.1 & P.Féd.2 & P.Pr. & $\begin{array}{l}\text { Moyenne } \\
\text { sur les } \\
4 \text { sites }\end{array}$ \\
\hline $\begin{array}{l}\text { Codt de production } \\
\text { (ch. d'exploitation + ch. financieres) }\end{array}$ & 29250 & 16650 & 18000 & 12550 & 19100 \\
\hline Production de brochetons/ha terrain/an & 14250 & 11300 & 12900 & 6800 & 11300 \\
\hline Prix de revient du brocheton & 2,0 & 1,5 & 1,4 & 1,8 & 1.7 \\
\hline
\end{tabular}

\section{Produit d'exploitation}

Le produit d'exploitation varie de 24.000 à $50.000 \mathrm{~F} / \mathrm{ha}$ de terrain/an, selon les ésocicultures, avec une moyenne avoisinant $40.000 \mathrm{~F} / \mathrm{ha}$ de terrain/an (Tableau V).

Cette fluctuation importante du produit d'exploitation est due à la variabilité inter-sites des paramètres suivants:

- la part de terrain effectivement aménagée en étangs (de 46 à $71 \%$; Tableau 1);

- le niveau de production (de 1,4 à 2,8 brochetons $/ \mathrm{m}^{2}$; Tableau I).

La superficie relative en eau est avant tout fonction de la taille des étangs : elle augmente de moitié lorsque l'on passe d'une batterie d'étangs de 400-900 $\mathrm{m}^{2}$ (P.Féd.2) à un lot d'étangs de 2.500-3.000 $\mathrm{m}^{2}$ (P.Féd.1).D'autre part, elle dépend de la largeur des digues : une largeur de digue relativement importante (de 6 à $8 \mathrm{~m}$ ) peut être justifiée par la nécessité de permettre le passage des véhicules intervenant lors des travaux de réaménagement et/ou par la présence sur le site de rongeurs constructeurs de galeries.

Le niveau de production varie, en premier lieu, selon les caractéristiques de chaque site en tant que milieu de survie pour les embryons et les larves, et de prégrossissement pour les juvéniles (richesse trophique). Il dépend également du stade de récolte choisi: la densité numérique en juvéniles décroît de façon continue au cours de l'élevage extensif, selon des pentes variables avecle site et la période de croissance considérés (Bry et al., données non publiées).

Le stade 2-2,5 g (7,5-8 cm) a été adopté par un certain nombre d'ésocicultures (par exemple, le Paraclet et P.Féd.1) car il permet généralement d'obtenir à la fois des effectifs et une taille satisfaisants pour les opérations ultérieures d'introduction en milieu naturel (Bry, données non publiées). La forte densité numérique $\left(2,8\right.$ brochetons $\left./ \mathrm{m}^{2}\right)$ enregistrée par P.Féd.2 est en partie vraisemblablement liée au stade relativement précoce $(1,3 \mathrm{~g})$ de récolte.

II convient enfin de remarquer que les niveaux de production rapportés dans cette étude ne peuvent pas être considérés de façon définitive comme caractéristiques des différents sites (à l'exception du Paraclet), le nombre d'étangs et/ou d'années étudiés étant limité. Les niveaux de production mentionnés pour P.Féd.1, P.Féd. 2 et P.Pr. sont cependant en accord avec la gamme de valeurs classiquement obtenue dans ce type d'élevage, et ont donc été retenus pour l'évaluation du produit d'exploitation de ces trois établissements. 
Il faut noter, par ailleurs, que le prix de vente du brocheton, fixé par souci de simplification à $3,50 \mathrm{FH} . \mathrm{T}$. livré, est en fait variable selon les régions (de 2,50 à 4,50 FH.T. livré). Cette variabilité peut influer de façon importante sur le produit d'exploitation.

\begin{tabular}{|c|c|c|c|c|c|c|}
\hline Table V & \multicolumn{6}{|c|}{$\begin{array}{l}\text { : Net sales, operating profit and farm income (in F.F. 1986/ha of land/year, exclusive } \\
\text { of tax). }\end{array}$} \\
\hline Sites & & Paraclet & P.Féd.1 & P.Féd. 2 & P.Pr. & $\begin{array}{l}\text { Moyenne } \\
\text { sur les } \\
4 \text { sites }\end{array}$ \\
\hline \multicolumn{2}{|c|}{ Produit d'exploitation" } & 49850 & 39650 & 45250 & 23850 & 39650 \\
\hline \multicolumn{2}{|c|}{ Charges d'exploitation } & 16300 & 8950 & 9950 & 9100 & 11050 \\
\hline \multicolumn{2}{|c|}{$\begin{array}{l}\text { Resultat d'exploitation } \\
(=\text { prodult - charges d'exploitation) }\end{array}$} & 33550 & 30700 & 35300 & 14750 & 28600 \\
\hline \multicolumn{2}{|c|}{ Charges financières } & 12950 & 7700 & 8050 & 3450 & 8050 \\
\hline \multicolumn{2}{|c|}{$\begin{array}{l}\text { Revenu agricole } \\
\text { (= résultat d'expl. - charges financ.) }\end{array}$} & 20600 & 23000 & 27250 & 11300 & 20550 \\
\hline
\end{tabular}

- Prlx de vente du brocheton de $7 / 8 \mathrm{~cm}=3,50 \mathrm{~F}$ H.T. livré.

\section{Rentabilité de la R.N.A.}

Le résultat d'exploitation varie, selon les piscicultures étudiées, d'environ 15.000 à 35.000 F/ha de terrain/an (moyenne : $29.000 \mathrm{~F} /$ ha de terrain/an; Tableau $\mathrm{V}$ ).

La rentabilité de la R.N.A. est traduite par le revenu agricole, égal au résultat d'exploitation diminué des frais financiers (Tableau V). Celui-ci varie, selon les sites, de 11.000 à $27.000 \mathrm{~F} / \mathrm{ha}$ de terrain/an (moyenne : $20.500 \mathrm{~F} /$ ha de terrain/an).

Malgré cette importante variabilité, la rentabilité de la R.N.A. demeure très élevée - revenu agricole moyen $=52 \%$ du produit d'exploitation (Figure 2) - si l'on sait que peu d'entreprises dépassent $10 \%$ de rentabilité avant impôt (Jaclin, 1983). II convient cependant de nuancer les résultats rapportés dans le Tableau 5 , la production de brochetons pouvant en effet constituer une activité intéressante mais annexe, plutôt qu'une entreprise à part entière.

N.B. : II faut signaler, en outre, que les jeunes pisciculteurs peuvent bénéficier, à leur installation et sous certaines conditions, d'un abattement de $50 \%$ sur le revenu imposable pendant cinq ans.

\section{Comparaison des performances économiques de la R.N.A. avec celles d'autres types d'élevage et de culture}

\subsection{Pisciculture en étangs/R.N.A.}

II n'existe actuellement que peu de données économiques disponibles relatives à la pisciculture en étangs.

Dans le cadre d'une étude sur les potentialités de l'aquaculture dans la région Rhône-Alpes, Guerrin, Vallod et Gault (1984) rapportent qu'en élevage extensif classique (production de carpes principalement, ainsi que de tanches, poissons blancs et brochets), les bénéfices d'exploitation suivants - directement comparables aux résultats d'exploitation, hors impôts fonciers, mesurés dans le présent article - peuvent être escomptés:

— dans la Dombes (Ain) : $1.000 \mathrm{~F} / \mathrm{ha}$ d'étang/an, en 1983 (pour un rendement annuel de $250 \mathrm{~kg} / \mathrm{ha}$ ), soit un peu moins de 1.200 F 1986/ha d'étang/an;

- dans le Forez (Massif Central) : de 1.500 à $2.000 \mathrm{~F} / \mathrm{ha}$ d'étang/an, en 1983 (pour un rendement annuel de $360 \mathrm{~kg} / \mathrm{ha}$ ), soit de 1.750 à $2.350 \mathrm{~F}$ 1986/ha d'étang/an.

En R.N.A., le résultat d'exploitation - hors impôt foncier - varie, selon les ésocicultures

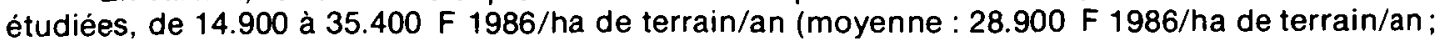
terrain = étangs + digues), soit 6 à 30 fois celui obtenu en pisciculture en étangs.

L'ésociculture se caractérise ainsi par une structure composée d'un nombre limité de petits étangs et par une rentabilité intéressante (en particulier dans le cas de la R.N.A.), compte tenu notamment des prix de vente élevés des juvéniles produits. Cette activité ne saurait, cependant, 


\section{PARACLET}

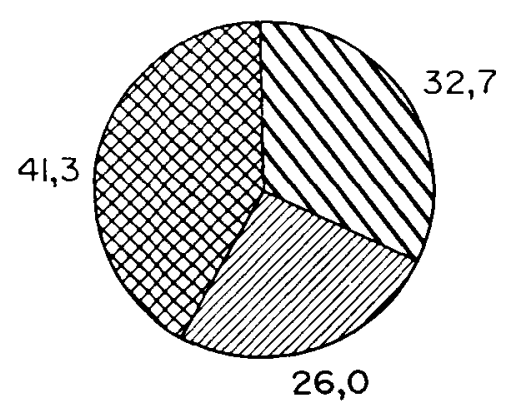

MOYENNE SUR LES 4 SITES

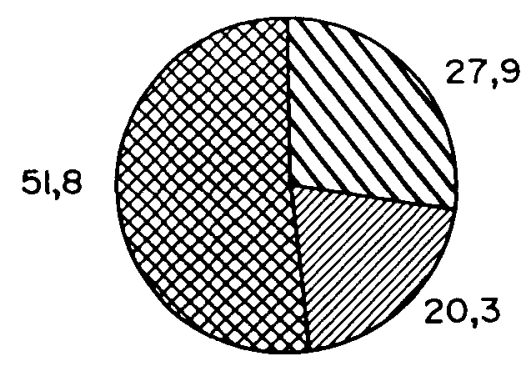

P. FED. 2

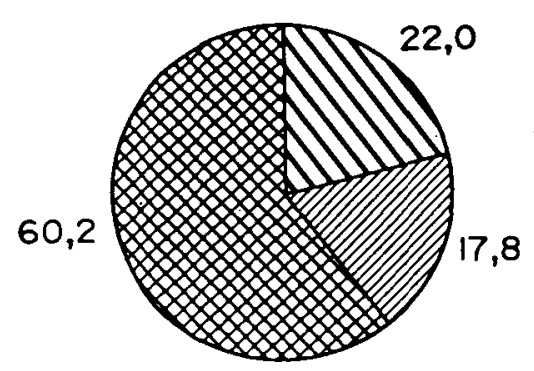

P. FED. I

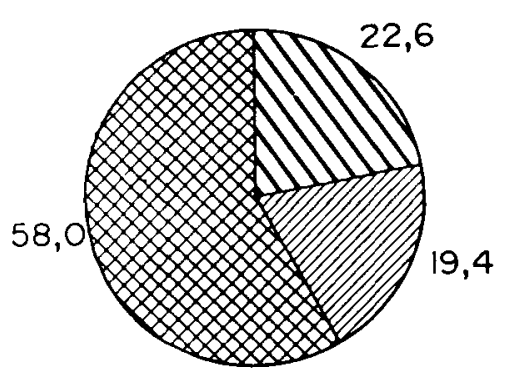

DVE

EI

RA

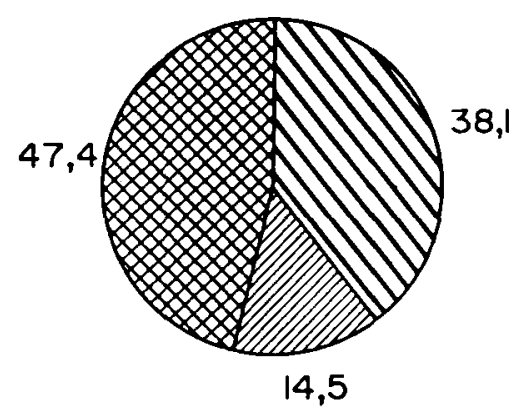

Figure 2 : Charges d'exploitation, charges financières et revenu agricole exprimés en \% du produit d'exploitation.

Abréviations utilisées:

- EX : Charges d'exploitation

- FI : Charges financières

- RA : Revenu agricole.

Figure 2 : Representation of operating expens2s, financial charges and farm income in \% of the net sales.

- EX : Operating expenses

- FI : Financial charges

- RA : Farm income. 
s'envisager sur des superficies totales comparables à celles des étangs de cypriniculture, la finalité et le marché des produits de ces deux types de pisciculture étant fondamentalement différents.

\subsection{Populiculture/R.N.A.}

Le peuplier est une essence souvent utilisée pour valoriser certaines parcelles agricoles humides, impropres aux cultures classiques, ces mêmes parcelles pouvant, dans certaines conditions, convenir à l'aménagement d'une ésociculture.

Le revenu agricole obtenu en populiculture, au bout de 20 à 25 ans, est en moyenne, à l'heure actuelle, en Picardie (première région productrice de peupliers en France), de l'ordre de $1.600 \mathrm{~F} / \mathrm{ha} / \mathrm{an}$, représentant $76 \%$ du produit d'exploitation (données du Centre Régional de la Propriété Forestière d'Amiens). II faut souligner, par ailleurs, que le cours du bois est généralement fluctuant et imprévisible.

Le revenu agricole obtenu en R.N.A., dès la première année d'exploitation, est en moyenne, sur les deux ésocicultures picardes étudiées (Paraclet et P.Pr.), de $16.000 \mathrm{~F} /$ ha de terrain/an (44\% du produit d'exploitation).

\subsection{Cultures et élevages agricoles traditionnels/R.N.A.}

Le revenu agricole calculé sur un échantillon de 1.331 exploitations agricoles de la Somme s'élève, toutes productions confondues, à un peu plus de $1.800 \mathrm{~F} / \mathrm{ha}$ de Surface Agricole Utile/an, soit $13 \%$ du produit d'exploitation (données 1986 du Centre Départemental d'Économie Rurale et de Gestion de la Somme).

Le revenu agricole, en R.N.A., est en moyenne, sur les deux ésocicultures de la Somme (Paraclet et P.Pr.), de $16.000 \mathrm{~F} / \mathrm{ha}$ de terrain/an (44\% du produit d'exploitation).

La comparaison de la R.N.A. avec l'agriculture classique n'est faite ici qu'à titre indicatif, ces deux systèmes n'étant pas réellement comparables:

- Les superficies concernées et, généralement, les terrains utilisés, ne sont pas les mêmes dans les deux cas:

- Les cultures et élevages agricoles traditionnels font l'objet d'un marché ancien et structuré, alors que le marché du poisson de repeuplement est souvent difficile à évaluer avec précision.

\section{CONCLUSION}

La création d'une ésociculture est soumise aux conditions préalables suivantes :

- nécessité d'une étude de marché du brocheton de repeuplement, la situation étant variable d'un département à l'autre (Williot, 1980);

- autorisation de création d'un enclos piscicole, délivrée par la Direction Départementale de l'Agriculture.

Bien que reposant sur seulement quatre cas, la présente analyse économique devrait permettre d'évaluer convenablement la rentabilité de la R.N.A., compte tenu notamment de l'hétérogénéité de comportement des investisseurs considérés ici (Tableau III).

Elle fait apparaître qu'un revenu agricole de 11.000 à $27.000 \mathrm{~F} /$ ha de terrain/an (en moyenne 20.000 F/ha de terrain/an) peut être obtenu.

Cette rentabilité élevée de la R.N.A., associée à sa fiabilité technique, permet d'envisager raisonnablement un développement prochain de son application:

- au niveau des Fédérations Départementales de Pêche et de Pisciculture, et de certaines piscicultures en étangs, en tant que méthode simple de production de brochetons de repeuplement de qualité (Bry et al., 1984), demandant des investissements pouvant se limiter au seul aménagement des étangs;

- au sein d'exploitations agricoles, en tant que source de revenus complémentaires, permettant également de valoriser des terres à faible potentialité et de diversifier la production.

Il convient finalement d'insister sur le fait que les surfaces consacrées à un élevage extensif de brochetons se limitent généralement à quelques hectares, pour différentes raisons:

- limites naturelles de l'étendue des sites favorables;

- limites du marché local;

- difficultés pratiques de livraison immédiate d'une production concentrée sur une dizaine de jours:

- normes de mise en charge recommandées pour l'introduction des juvéniles en milieu naturel (densités de peuplement modérées). 


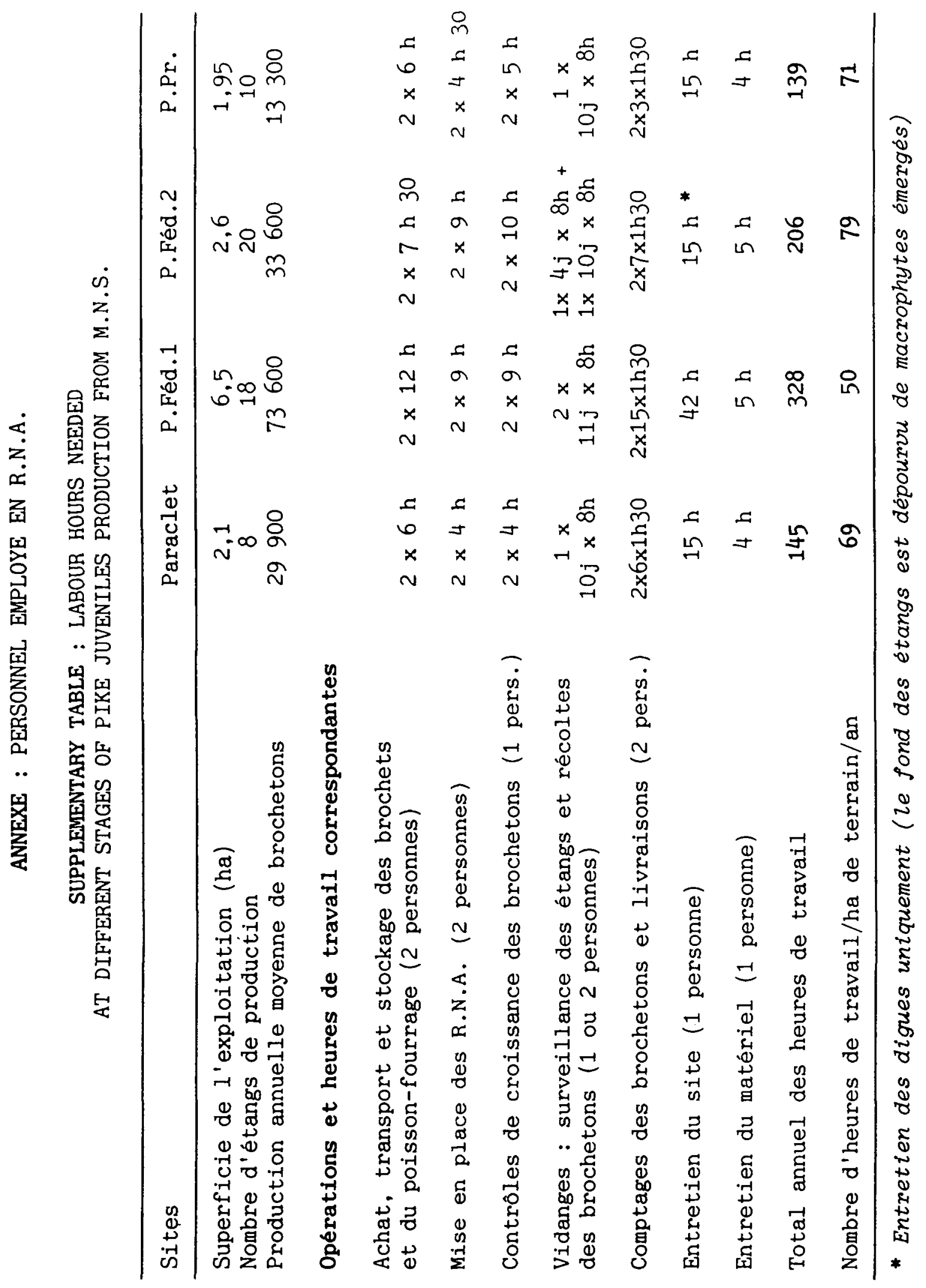




\section{REMERCIEMENTS}

Ce travail a été réalisé dans le cadre d'une convention CSP/INRA référence 85327 et s'intègre dans une thèse de Doctorat (Institut National Polytechnique de Toulouse).

Les auteurs tiennent à remercier, pour leur aide à la réalisation de ce travail, Messieurs JANTZEN J.M., SERY F. et NEVEU G. (Conseil supérieur de la Pêche, Centre du Paraclet), REVET B. (Entrepreneur à Bettencourt-Saint-Ouen, Somme), THULLIEZ P. (C.D.E.R.G. de Doullens, Somme), NIHOUARN (Délégation Régionale du C.S.P. Bretagne-Basse Normandie), GILLY B. (I.F.R.E.M.E.R. Paris), LANGUILLE (Professeur d'Économie au Lycée Agricole du Paraclet), VAN DE VOORDE M. (S.R.A.E. Picardie à Amiens), FICHEUX (Centre des Impôts Fonciers d'Amiens, Somme), POLIAUTRE (Centre Régional de la Propriété Forestière d'Amiens Somme), LOBIT F. (I.N.S.E.E. Amiens, Somme), ainsi que les Présidents et les Responsables Techniques des Fédérations et piscicultures citées dans l'étude.

Nous remercions également Mesdemoiselles JARRY B. et DAVID M.A., pour la réalisation informatique de cet article.

\section{RÉFÉRENCES}

BRY C., SOUCHON Y., 1982. Production of young northern pike families in small ponds: natural spawning versus fry stocking. Trans. am. Fish. Soc., 111, 476-480.

BRY C., SOUCHON Y., NEVEU G., TREBAOL L., 1983. Production de familles de brochetons en petits étangs par reproduction naturelle aménagée: bilan de trois années d'expérimentation et comparaison avec la méthode d'alevinage, p. 63-73. In Billard R., Ed. Le Brochet: gestion dans le milieu naturel et élevage, I.N.R.A. Publ., Paris.

BRY C., SOUCHON Y., NEVEU G., 1984. Note technique : Production de brochetons en petits étangs par reproduction naturelle aménagée. Bull. Fr. Pisçic., 293-294, 59-64.

GUERRIN F., VALLOD D., GAULT J., 1984. Potentialités de l'aquaculture en région Rhône-Alpes. Étude réalisée par la Division Aménagements littoraux et Aquaculture du C.E.M.A.G.R.E.F. Montpellier, et par le Laboratoire d'Ichtyologie des Facultés Catholiques de Lyon, 169 p.

JACLIN F., 1983. Le Guide de la Création d'Entreprise, Collection Connaître et Pratiquer la Gestion. Ed. F. Nathan, Paris, 224 p.

LEFEBVRE F., 1987. Memento pratique : Agriculture (fiscal, social, comptable, juridique), Ed. F. Lefebvre, Paris, $1134 \mathrm{p}$.

WILLIOT P., 1980. Etat actuel de la pisciculture d'étang en France, p. 411-425. In Billard R., Ed. La pisciculture en étang, I.N.R.A. Publ., Paris. 